\title{
Evaluación del estado micorrícico de plántulas de Pinus ponderosa producidas bajo fertirriego, sin manejo de la micorrización
}

\author{
Evaluation of the mycorrhizal condition of ponderosa pine seedlings produced \\ with high fertilization levels and lacking mycorrhizal management
}

\author{
María Eugenia Salgado Salomónn ${ }^{\mathrm{a}, \mathrm{c} *}$, Mario Rajehenberga,b,c, Carolina Barroetaveñaa ${ }^{\mathrm{a}, \mathrm{b}, \mathrm{c}}$ \\ *Autor de correspondencia: aUniversidad Nacional de la Patagonia "San Juan Bosco", Sarmiento 849, CP 9200, Esquel, \\ Chubut, Argentina, tel.: 54-2945-450820, mesalgadosalomon@gmail.com \\ bCentro de Investigación y Extensión Forestal Andino Patagónico, CIEFAP, Argentina, \\ ${ }^{\mathrm{c} C o n s e j o ~ N a c i o n a l ~ d e ~ I n v e s t i g a c i o n e s ~ C i e n t i ́ f i c a s ~ y ~ T e c n o l o ́ g i c a s, ~ C O N I C E T, ~ A r g e n t i n a . ~}$
}

\begin{abstract}
SUMMARY
Several reports of tree planters from the Patagonian Andes region of Chubut (Argentina) have pointed out that seedlings of Pinus ponderosa, obligate ectotrophic species, produced in greenhouses with high levels of fertilization without ectomycorrhizal inoculum applications, showed low or null ectomycorrhizal infection at the end of nursery stage but had excellent performance in plantation sites during the first years. This study was planned to evaluate if those seedlings develop mycorrhizal associations once installed in plantations, and if those associations are originated from inoculums acquired in the nursery. Three evaluations were done: 1) analyses of seedlings at the end of nursery period, to evaluate their initial mycorrhizal condition, 2) analyses after 6 month, with seedlings transplanted into pots with semi-sterile substrate, to establish if there exists inoculum in the system that did not express because of the high fertilization levels and, 3) analyses after 24 month, with seedlings planted in a field distant from any source of ectomycorrhizal inoculum, to evaluate the associations developed in the planting site. Results showed that: 1) the studied nursery produced seedlings with null or very low levels of mycorrhization, with low morphotypes' richness; 2) seedlings showed very high mycorrhizal percentages after 6 months (under greenhouse conditions) and after 24 months (in field conditions); 3) different mycorrhizal fungi behave differently under fertilization. The analyzed nursery fortuitously incorporated mycorrhizal inoculum that rapidly colonized seedlings, immediately after fertilization was interrupted. This fact demonstrates that the hypothesis that ponderosa pine seedlings can establish and grow in anectotrophic environments without EM symbioses is false.
\end{abstract}

Key words: inoculum, nursery, mycorrhizas, Patagonia.

\section{RESUMEN}

Repetidos reportes de plantadores de la zona cordillerana del Chubut (Argentina) han señalado que las plántulas de Pinus ponderosa, especie ectotrófica obligada, producidas bajo invernáculo con fertirriego y sin aplicación de inoculantes ectomicorrícicos, que presentaban baja o nula infección ectomicorrícica al terminar la etapa de vivero, mostraron excelentes comportamientos en los sitios de plantación durante los primeros años. Por ello, se planteó este estudio con el objetivo de constatar si las plántulas allí producidas desarrollan asociaciones micorrícicas una vez llevadas a plantación, y si éstas se originan a partir de inóculo adquirido en el vivero. Para ello, se realizaron tres evaluaciones de las plántulas: 1) al salir del vivero de producción acelerada, para constatar el estado micorrícico inicial, 2) después de seis meses de crecimiento luego de un trasplante a macetas con sustrato semi-estéril, para constatar si existía inóculo en el sistema que no se expresaba por el fertirriego, y 3) después de 24 meses luego de implantadas en un predio alejado de cualquier fuente de inóculo ectomicorrícico, para constatar el tipo de asociaciones formadas en el sitio de plantación. Los resultados obtenidos permitieron demostrar que: 1) el vivero de producción acelerada produjo plántulas con nulo o muy bajo porcentaje de micorrización y una baja riqueza en morfotipos; 2) las plántulas presentaron muy buenos porcentajes de micorrización al cabo de seis meses (en condiciones de invernáculo de crecimiento) o de dos años (en condiciones de campo), y 3) los diferentes hongos micorrícicos reaccionaron diferencialmente a la aplicación de fertirriego. Se evidenció que el vivero analizado incorpora fortuitamente inóculo micorrícico que coloniza rápidamente a las plántulas inmediatamente después de suspendida la fertirrigación, demostrando que la hipótesis de que las plántulas de $P$. ponderosa pueden establecerse y crecer en ambientes anectotróficos sin asociaciones EM es falsa.

Palabras clave: inóculo, vivero, micorrizas, Patagonia. 


\section{INTRODUCCIÓN}

La producción de plántulas forestales de coníferas de rápido crecimiento bajo un sistema de "dieta" o fertirriego propone una mejora económica pues, al acelerar la tasa de crecimiento de los plantines, acorta la duración de la etapa de vivero. Estas plántulas producidas en contenedores con sustrato artificial, bajo invernáculo con temperatura controlada y con aplicación de una dieta apropiada de fertilizantes (fertirriego) muestran un crecimiento óptimo (Gagnon et al. 1987).

Las coníferas, y en particular el género Pinus, necesitan establecer asociaciones simbióticas con hongos ectomicorrícicos para proveerse de nutrientes esenciales, principalmente nitrógeno, fósforo y agua (Meyer 1973, Read et al. 1992, Smith y Read 2008). Numerosos estudios han demostrado la superioridad de las plántulas micorrizadas frente a las no micorrizadas en términos de establecimiento y supervivencia en campo, generando mejores respuestas a los crecimientos en las forestaciones (Gagnon et al. 1988 y referencias allí incluidas, Stenström et al. 1990, 1997, Krasowski et al. 1999, Jumpponen 2001, Van Tichelen et al. 2001, Dunabeitia et al. 2004, Ortega et al. 2004). Sin embargo, el manejo de la micorrización bajo sistemas que reciben fertilización requiere un estudio detallado para cada caso, dado que existen reportes contradictorios sobre el efecto inhibidor de la fertilización sobre la micorrización, dependiendo de las especies arbóreas y fúngicas involucradas (Menkis et al. 2005).

Existen tres posiciones discordantes entre distintos autores respecto al efecto de la fertilización sobre la micorrización de plántulas en viveros. Algunos han reportado que altos niveles de fertilizantes inhiben la micorrización que, en caso de existir, resulta muy pobre (Gagnon et al. 1987, 1988, Hunt 1988, Reitveld et al. 1989, Chakravarty y Chatarpaul 1990, Le Tacon et al. 1997, Smith y Read 1997). Otros, en cambio, han reportado que la formación de micorrizas sería independiente de la fertilización, pues no encontraron diferencias entre tratamientos con distintas dosis de fertilizantes (Molina y Chamard 1983, Danielson et al. 1984, Khasa et al. 2001). Trappe (1977), por su parte, reconocía que cada hongo ectomicorrícico reacciona de manera diferente frente a la fertilización, aumentando la micorrización en algunos casos o disminuyéndola drásticamente en otros, lo cual explicaría los resultados discordantes entre diferentes estudios.

Información recibida de los plantadores de la zona cordillerana del Chubut (Argentina) señalaba que las plántulas de pino ponderosa (Pinus ponderosa Dougl. ex Laws.) producidas bajo invernáculo con fertirriego, sin aplicación de inoculantes ectomicorrícicos (EM), y con baja o nula infección natural al terminar la etapa de vivero, tal como fue verificado por los autores como parte de este trabajo, habían tenido un excelente comportamiento en el campo en los primeros años posteriores a la plantación ${ }^{1}$. Esta situación planteó tres hipótesis: 1) que existe alguna fuente de inóculo ectomicorrícico en el sistema y que la colonización no se expresa por el uso de fertilizantes. Así, las plantas tendrían inóculo disponible que produciría la infección una vez en el sitio de plantación; 2) que las plántulas no desarrollan asociaciones micorrícicas; y 3) que las plántulas desarrollan asociaciones EM con inóculo presente en el sitio de plantación.

Dado que las EM son altamente específicas (Smith y Read 1997) y que los pastizales y subarbustos del ecotono bosque-estepa patagónicos carecen de asociaciones EM, formando asociaciones típicamente endomicorrícicas (Godoy et al. 1994, Fontenla et al. 1998, 2001), la tercera hipótesis parecía poco factible, especialmente cuando las plántulas son llevadas a sitios carentes o alejados de cualquier otra plantación que pudiera actuar como fuente de inóculo EM. La segunda hipótesis también parecía poco probable, ya que los sitios que se forestan con pino ponderosa poseen deficiencias estacionales marcadas en la disponibilidad de agua, heladas reiteradas e intensas, situaciones difíciles de resistir para una plántula desprovista de la simbiosis (Le Tacon et al. 1997). La hipótesis 1 era la que parecía más lógica considerando la fisiología de la especie, dependiente de asociaciones EM, y las condiciones de los sitios de plantación (Read et al. 1992, Simard et al. 2002, Smith y Read 2008).

El Programa de Apoyo a la Investigación y Desarrollo Regional Forestal (PAIDER Forestal) de la Facultad de Ingeniería, Universidad Nacional de la Patagonia "San Juan Bosco" (UNPSJB), sede Esquel (Chubut, Argentina), fue concebido como un modelo de producción y transferencia tecnológica en la región, produciendo plántulas forestales bajo sistema de fertirriego desde el año 2000. Dado que las experiencias que desde allí surgen son tomadas como referencia para la región, se planteó como objetivo de este trabajo constatar si las plantas allí producidas desarrollan asociaciones micorrícicas en plantación, y si éstas provenían de inóculo adquirido en el vivero.

\section{MÉTODOS}

El estudio se realizó con plántulas de ocho meses del vivero de producción acelerada de plántulas forestales del PAIDER de la UNPSJB, Esquel, Chubut, Argentina. Las mismas se cultivaron a partir de semillas de calidad comercial provenientes de EEA INTA Trevelin (Chubut, Argentina) sobre sustrato inerte (50\% turba $+50 \%$ arena volcánica) y fueron regadas con agua de perforación propia del productor. Se muestreó el vivero extrayendo 240 ejem-

\footnotetext{
Juan Enricci, responsable del Programa de Apoyo a la Investigación y Desarrollo Regional Forestal (PAIDER Forestal), Universidad Nacional de la Patagonia "San Juan Bosco", Esquel, Chubut, Argentina. Comunicación personal.
} 
plares con características morfológicas homogéneas. Las mismas se asignaron al azar a tres grupos o tratamientos de 80 ejemplares cada uno.

- Tratamiento 1: fue evaluado inmediatamente después de extraído del vivero de producción acelerada con el fin de analizar el estado micorrícico inicial de las plántulas.

- Tratamiento 2: se trasplantó en macetas de $150 \mathrm{~cm}^{3}$ con sustrato estéril [constituido por proporciones iguales de arena volcánica esterilizada en estufa a $100^{\circ} \mathrm{C}$ durante $72 \mathrm{~h}$ y suelo tamizado (Parque Nacional Los Alerces, $\left.42^{\circ} 59^{\prime} 18,5^{\prime \prime} \mathrm{S}, 71^{\circ} 28^{\prime} 46^{\prime \prime} \mathrm{O}\right)$ y tindalizado tres veces consecutivas a lo largo de tres días durante 1,5 h cada uno]. Las macetas se mantuvieron seis meses en un invernáculo de crecimiento, diferente al de producción acelerada, alejado de cualquier posible fuente natural de inóculo micorrícico, asegurándoles condiciones apropiadas de humedad con riegos regulares con agua corriente, sin fertilizantes, proveniente de un pozo diferente y alejado del que provee al vivero de producción acelerada.

- Tratamiento 3: se llevó inmediatamente a plantación (42 ${ }^{\circ}$ 54' 47' S, 71 15 ' 30" O, Esquel, Chubut) en un pastizal sin plantaciones forestales cercanas (a más de $5 \mathrm{~km}$ ) que pudieran servir de fuente de inóculo ectomicorrícico. Las plántulas se dispusieron en un sector homogéneo, con un espaciamiento de 3 x $3 \mathrm{~m}$, y fueron descalzadas y evaluadas a los 24 meses.

Las plántulas de los tres tratamientos fueron evaluadas en los momentos de crecimiento radicular (en otoño para el tratamiento 1 , y en primavera para los tratamientos 2 y 3) cuando la colonización micorrícica también está en plena actividad (Smith y Read 2008).

En todos los sistemas radicales se evaluó la presencia de ecto- y ectendomicorrizas. Para ello se consideraron la calidad de micorrización, la riqueza de morfotipos micorrícicos y la composición en morfotipos.

La calidad de micorrización se expresó como: a) el porcentaje de micorrización según la metodología descrita por Brundrett et al. (1996), evaluando la cantidad de puntas micorrizadas a lo largo de las raíces. En los tratamientos 1 y 2 se evaluaron $150 \mathrm{~cm}$ de raicillas del sistema radical. En el tratamiento 3, que poseía un sistema radical más fibroso, se tomaron $200 \mathrm{~cm}$ de las raicillas con el fin de disminuir el error de muestreo. El porcentaje de micorrización total (PM) se calculó sobre el total de la muestra obtenida:

$$
\mathrm{PM}=(\text { puntas micorrizadas/total de puntas }) \times 100
$$

b) la cantidad de puntas micorrizadas por largo de raíz (PM/mR), de acuerdo con Brundrett et al. (1996):

$\mathrm{PM} / \mathrm{mR}=$ puntas micorrizadas/longitud de la raíz (m) [2]
La riqueza de morfotipos se expresó como: a) riqueza total de morfotipos, calculada como el número de morfotipos diferentes presentes en cada tratamiento; y b) riqueza promedio de morfotipos, calculada como el promedio de morfotipos diferentes encontrados en todas las plántulas de cada uno de los tratamientos.

La composición en morfotipos se expresó como la cantidad de plántulas que poseían los diferentes morfotipos en cada tratamiento.

Los morfotipos y los hongos micorrícicos que los formaban se determinaron realizando observaciones del sistema radical en agua bajo lupa Wild M32. Se observaron características morfológicas del tamaño, color, arquitectura, presencia de hifas remanentes y rizomorfos según metodología descrita por Goodman et al. (1996). Ante la presencia de morfotipos no descritos previamente (Barroetaveña 2004, Barroetaveña et al. 2005, Barroetaveña et al. 2007), se realizaron preparados microscópicos en agua para evaluar las microestructuras: características de la red de Hartig y del manto, forma y dimensiones de las hifas, presencia y tipo de septos y concreciones de cristales sobre las paredes u ornamentaciones (Agerer 1994, 2001).

Para una mejor sistematización de las observaciones se utilizaron las siguientes denominaciones para aquellos morfotipos que no se correspondían con hongos micorrícicos determinados taxonómicamente:

"Micelio blanco": ectomicorriza dicotómica (dos dicotomías) con manto emanante blanco abundante y algodonoso.

"E-strain I": ectendomicorriza simple, no ramificada, castaño clara, sin presencia de hifas externas.

"E-strain III": ectendomicorriza simpodial, engrosada en la punta, castaño clara, sin presencia de hifas externas.

"E-strain IV": ectendomicorriza pinnada, ligeramente engrosada en la punta, castaño clara, sin presencia de hifas externas.

"Simple": ectomicorriza simple, con micelio emanante blanco, escaso, de aspecto estrigoso.

"Micelio marrón": ectomicorriza simple, ligeramente engrosada en la punta, con micelio emanante castaño abundante, de aspecto estrigoso.

"Amarilla": ectomicorriza dicotómica a simpodial, engrosada en la punta, amarilla, con micelio blanco nacarado, abundante, formando parches.

Se consideraron "puntas muertas" a aquellas que presentaron la peridermis colapsada, sin posibilidad de identificar correctamente las microestructuras ni las características macroscópicas de la misma.

El análisis de PM, PM/mR y riqueza de morfotipos entre distintos tratamientos se realizó mediante la prueba de Kruskal-Wallis, un modelo no paramétrico, y posteriores comparaciones múltiples con la prueba de Mann-Whitney $(P \leq 0,05)$ (Steel y Torrie 1988).

Para analizar las semejanzas en la composición en morfotipos entre los distintos tratamientos se utilizó un análisis de clusters jerárquicos, aplicando la prueba de 
Ward. Para ello se construyó una matriz con la composición en morfotipos (número de plantas de cada tratamiento que poseían cada morfotipo). Todos los análisis se realizaron utilizando el paquete estadístico SPSS para Windows, versión 11.5.

\section{RESULTADOS}

Porcentaje de micorrización. Los porcentajes de micorrización mostraron diferencias significativas (Kruskal-Wallis, $P<0,001$ ) entre el tratamiento 1 con el 2 y el 3 (MannWhitney, $P<0,001$ para ambos), pero no entre los tratamientos 2 y 3 (Mann-Whitney, $P=0,254$ ). Las PM/ $\mathrm{mR}$ mostraron diferencias significativas (Kruskal-Wallis, $P<0,001)$ entre los tres tratamientos (Mann-Whitney, $P<0,001$ en todos los casos).

El PM en el tratamiento 1 fue bajo. Una sola plántula presentó $71 \%$ de micorrización, en tanto que $66 \%$ de las plántulas no presentó micorrización (cuadro 1). Las restantes plántulas (33\%) presentaron un rango entre $1 \mathrm{y}$ $37 \%$. El promedio de puntas micorrizadas por metro de raíz también fue bajo (cuadro 1). El tratamiento 2 presentó un PM con valores de infección mayores pero muy variables, los que oscilaron entre 0 y $92,8 \%$. Las $\mathrm{PM} / \mathrm{mR}$ presentaron un comportamiento similar (cuadro 1). El tratamiento 3 presentó PM ligeramente mayor que el tratamiento 2, pero con una variación mucho menor (cuadro 1). Los valores oscilaron entre $29,4 \%$ y $94,6 \%$. Las PM/mR se comportaron de manera similar a las PM (cuadro 1).

Cuadro 1. Valores de porcentaje de micorrización (PM), puntas micorrizadas por metro de raíz $(\mathrm{PM} / \mathrm{mR})$ y riqueza de morfotipos obtenidos en los tres tratamientos.

Values of mycorrhization percentages (PM), mycorrhized tips per root meter $(\mathrm{PM} / \mathrm{mR})$ and morphotypes richness from the bioassays.

\begin{tabular}{lccc}
\hline \multirow{2}{*}{ Característica } & \multicolumn{3}{c}{ Tratamiento } \\
\cline { 2 - 4 } & 1 & 2 & 3 \\
\hline Cantidad plantas & 80 & 80 & 73 \\
PM (\%) & 2,6 & 56,6 & 56,6 \\
$\sigma$ & 10,1 & 25,8 & 13,6 \\
PM/mR & 5,4 & 176,4 & 92,9 \\
$\sigma$ & 19,2 & 101,8 & 40,3 \\
Riqueza total morfotipos & 8 & 12 & 13 \\
Riqueza promedio morfotipos & 0,5 & 2,5 & 3,9 \\
$\sigma$ & 0,9 & 1,2 & 1,0 \\
\hline
\end{tabular}

Riqueza de morfotipos. La riqueza total de morfotipos en el tratamiento 1 fue más baja que en los tratamientos 2 y 3 (cuadro 1). Asimismo la riqueza promedio de morfotipos (el promedio de morfotipos diferentes encontrados en cada plántula) siguió la misma tendencia, mostrando diferencias significativas (Kruskal-Wallis, $P<0,001$ ) entre los tres tratamientos (Mann-Whitney, $P<0,001$ en todos los casos), siendo el tratamiento 3 el de valor más alto (cuadro 1).

Se observaron tres grupos de hongos formadores de micorrizas. El primer grupo (Suillus, "micelio blanco", "micelio marrón" y "tipo Tuber") se caracterizó por presentar una baja presencia en todos los tratamientos. El segundo grupo (Rhizopogon, "E-strain I" y "E-strain III") estuvo formado por hongos que estuvieron presentes en todos los tratamientos, pero su presencia aumentó marcadamente en los tratamientos 2 y 3. Un tercer grupo (Amphinema, "E-strain IV", "amarilla" y "simple") estuvo constituido por aquellas asociaciones presentes sólo en los tratamientos 2 y 3. No se observaron puntas muertas en el tratamiento 1, pero sí en el tratamiento 2 , con un importante aumento en el tratamiento 3 (cuadro 2).

Cuadro 2. Composición en morfotipos observados en cada tratamiento.

Morphotypes richness composition observed in each treatment.

\begin{tabular}{lccc}
\hline \multirow{2}{*}{ Morfotipos } & \multicolumn{3}{c}{ Tratamiento } \\
\cline { 2 - 4 } & 1 & 2 & 3 \\
\hline Cantidad de plantines & 80 & 80 & 73 \\
Suillus sp. & $1-9$ & $1-9$ & $1-9$ \\
Rhizopogon sp. & $10-29$ & $60-79$ & $80-100$ \\
Amphinema sp. & nd & $1-9$ & $1-9$ \\
Micelio blanco & $1-9$ & $1-9$ & $1-9$ \\
E- strain III & $1-9$ & $30-59$ & $60-79$ \\
E-strain I & $1-9$ & $80-100$ & $80-100$ \\
Simple & nd & $1-9$ & $1-9$ \\
Tipo Tuber sp. & $1-9$ & $1-9$ & $10-29$ \\
Micelio marrón & $10-29$ & $10-29$ & $10-29$ \\
E- strain IV & nd & $30-59$ & $30-59$ \\
Amarilla & nd & nd & $60-79$ \\
Puntas inmaduras & $1-9$ & $1-9$ & $1-9$ \\
Puntas muertas & nd & $1-9$ & $10-29$ \\
\hline
\end{tabular}

nd: no detectado.

El análisis de las semejanzas en composición de morfotipos entre los tres tratamientos a través de clusters jerárquicos delimitó dos subgrupos, uno incluyendo a los tratamientos 2 y 3 , con una distancia de combinación de 2,5, y otro correspondiente al tratamiento 1 , con una distancia de combinación al anterior de 25 (cuadro 3, figura 1).

\section{Distancia de combinación de cluster}

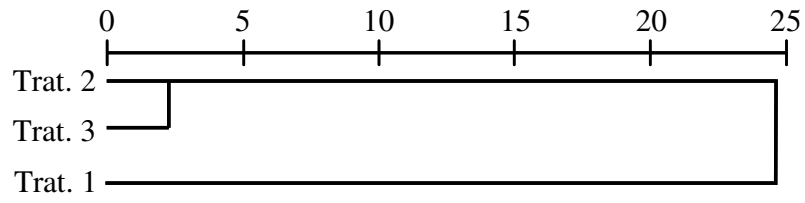

Figura 1. Dendrograma de la riqueza en morfotipos. Dendrogram of morphotypes richness. 
Cuadro 3. Descripción de los clusters formados por el estadístico (método de Ward).

Description of clusters formed by the Statistics (Ward's Method).

\begin{tabular}{|c|c|c|c|c|c|c|c|c|c|c|c|c|c|c|}
\hline \multicolumn{2}{|c|}{ Método de Ward* } & 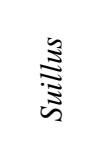 & $\begin{array}{l}\tilde{D} \\
\infty \\
0 \\
\tilde{D} \\
\tilde{\Xi}\end{array}$ & 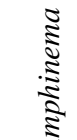 & 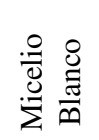 & 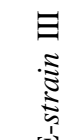 & 离 & 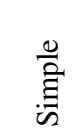 & $\begin{array}{l}\bar{\Xi} \\
\vdots \\
\vdots \\
\vdots\end{array}$ & 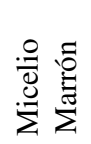 & 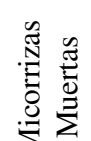 & 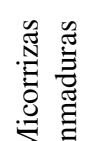 & 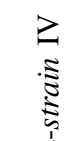 & 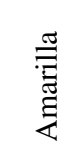 \\
\hline \multirow{3}{*}{1} & Media & 0,07 & 1,16 & 0,00 & 0,04 & 1,01 & 0,08 & 0,00 & 0,01 & 0,24 & 0,00 & 0,00 & 0,00 & 0,00 \\
\hline & $\mathrm{N}$ & 1 & 1 & 1 & 1 & 1 & 1 & 1 & 1 & 1 & 1 & 1 & 1 & 1 \\
\hline & $\sigma$ & 0,00 & 0,00 & 0,00 & 0,00 & 0,00 & 0,00 & 0,00 & 0,00 & 0,00 & 0,00 & 0,00 & 0,00 & 0,00 \\
\hline \multirow{3}{*}{2} & Media & 0,31 & 23,10 & 0,12 & 0,52 & 4,85 & 20,91 & 0,19 & 0,26 & 0,11 & 0,53 & 0,43 & 3,26 & 3,63 \\
\hline & $\mathrm{N}$ & 2 & 2 & 2 & 2 & 2 & 2 & 2 & 2 & 2 & 2 & 2 & 2 & 2 \\
\hline & $\sigma$ & 0,23 & 3,18 & 0,01 & 0,48 & 2,67 & 2,33 & 0,06 & 0,08 & 0,14 & 0,42 & 0,20 & 3,14 & 5,14 \\
\hline \multirow{3}{*}{3} & Media & 0,23 & 15,79 & 0,08 & 0,36 & 3,57 & 13,96 & 0,13 & 0,18 & 0,15 & 0,35 & 0,29 & 2,17 & 2,42 \\
\hline & $\mathrm{N}$ & 3 & 3 & 3 & 3 & 3 & 3 & 3 & 3 & 3 & 3 & 3 & 3 & 3 \\
\hline & $\sigma$ & 0,21 & 2,87 & 0,07 & 0,44 & 2,91 & 2,14 & 0,12 & 0,16 & 0,13 & 0,42 & 0,29 & 2,91 & 4,20 \\
\hline
\end{tabular}

*1: cluster 1; 2: cluster 2; 3: cluster 3. N: número de tratamientos que corresponden al cluster.

\section{DISCUSIÓN}

La presencia de plántulas micorrizadas en el tratamiento 1 permite afirmar que existe una fuente de inóculo micorrícico proveniente del vivero de producción acelerada. Las posibles fuentes de inóculo podrían ser cuatro: 1) El sustrato (arena volcánica), que no es esterilizado antes de la siembra y que se almacena debajo de ejemplares de pino ponderosa presentes en el predio del vivero, donde se han observado ocasionalmente fructificaciones de hongos micorrícicos. 2) Aporte aéreo de esporas provenientes de un vivero de coníferas a raíz desnuda ubicado a escasos $200 \mathrm{~m}$ del invernáculo y de una pequeña plantación de pino ponderosa cercana. 3) El agua utilizada para el fertirriego, que se almacena en un tanque australiano sin protección alguna, ubicado a metros de la citada plantación y dentro del vivero a raíz desnuda, que también podría recibir y concentrar el inóculo. 4) La cobertura seminal, que no es esterilizada al momento de la siembra. Para el caso del tratamiento 2 las fuentes de inóculo micorrícico serían aquellas heredadas de las del tratamiento 1 , ya que resulta altamente improbable que hubiera un aporte adicional aéreo y/o por agua debido a que: a) el invernáculo de crecimiento se encontraba lejos del vivero a raíz desnuda y de la plantación asociada; y b) la fuente de agua de riego del vivero de crecimiento provenía directamente de un pozo, diferente al que alimenta el tanque australiano.

Al comparar los porcentajes de micorrización total entre los tratamientos 1 y 2 , se observa un valor significativamente inferior en el tratamiento 1. Esto sugiere que la dieta de fertilizante aplicada en el vivero de producción acelerada estaría inhibiendo el proceso de formación de las micorrizas, tal como lo reportaron Gagnon et al. $(1987,1988)$, Chakravarty y Chatarpaul (1990), Le Tacon et al. (1997) y Martínez et al. (2007). De esta manera, al momento de ser plantadas en el campo las plántulas tenían baja o nula micorrización. Sin embargo, el inóculo micorrícico adquirido en el vivero de producción acelerada no dejó de ser viable y al terminar el efecto del fertirriego se manifestó abundantemente, tal como lo demostró el porcentaje de micorrización del tratamiento 2.

Los valores de porcentaje de micorrización entre los tratamientos 2 y 3 fueron similares. En cambio las $\mathrm{PM} / \mathrm{mR}$ del tratamiento 2 duplicaron el valor del tratamiento 3. Estos resultados podrían deberse a que las condiciones de campo (más rigurosas en cuanto a la disponibilidad de agua y las heladas) produjeron muerte de raicillas, disminuyendo la densidad de puntas micorrizadas en el último. Además, el tratamiento 3 mostró entre el 10-29\% de puntas micorrizadas muertas, mientras que en el tratamiento 1 no fueron detectadas y en el tratamiento 2 no superaron el $9 \%$ (figura 1).

La riqueza de morfotipos en el tratamiento 1 fue significativamente más baja que en los tratamientos 2 y 3 . Si bien el tratamiento 1 fue evaluado en otoño y los tratamientos 2 y 3 en primavera, y no puede descartarse un efecto de la estacionalidad en la expresión de la riqueza, los morfotipos encontrados en el tratamiento 1 fueron un subgrupo de los morfotipos hallados en los otros tratamientos. El aumento de la riqueza en los tratamientos 2 y 3 podría explicarse basándose en lo planteado por Trappe (1977), quien señala que no todos los hongos formadores de micorrizas reaccionan de igual manera frente la fertilización, y que ésta inhibió la expresión de alguno de ellos. Así, habría un grupo de hongos (Suillus, "micelio blanco, "micelio marrón" y "tipo Tuber") que sería menos propenso a la inhibición con fertirriego, manteniendo siempre una baja presencia en las plántulas. Otro grupo (Rhizopogon, "E-strain I" y "E- strain III") estaría compuesto por hongos micorrícicos que sí son afectados por la fertirrigación y que, si bien no 
son totalmente inhibidos, al quitar el fertirriego aumentaron su presencia. Un tercer grupo (Amphinema, "E-strain IV", "amarilla" y "simple") estaría constituido por aquellas asociaciones presentes sólo en los tratamientos 2 y 3 , tratándose de hongos micorrícicos que son inhibidos por la fertirrigación, pero con propágulos viables en el sistema, que sólo se expresaron con la suspensión del fertirriego. En el caso particular de "amarilla", que sólo apareció en el tratamiento 3 y de manera abundante, se interpreta que esta asociación requirió más tiempo para expresarse.

Las micorrizas del tipo "E-strain" (ectendomicorrizas) presentaron bajos porcentajes de colonización en el tratamiento 1, aumentando marcadamente en los tratamientos 2 y 3 (figura 1). Estas micorrizas usualmente predominan en plantines de vivero y en sitios degradados (Mikola 1988, Danielson 1991); están mundialmente distribuidas en diversos ambientes, como suelos de vivero con alto $\mathrm{pH}$, suelos acidificados o contaminados con metales pesados en minas, bosques naturales y plantaciones, áreas urbanas, etc. (Danielson 1991, Yu et al. 2001); presentan capacidad para colonizar tempranamente a las plántulas (Mikola 1988, Barroetaveña 2004), y podrían estar jugando un rol como pioneras en la colonización e infección de plántulas de pino ponderosa en los primeros meses de la plantación, beneficiando a las plántulas hasta que las condiciones medioambientales fueran favorables para una colonización con EM más vigorosa.

De las ectomicorrizas encontradas, solamente el género Rhizopogon y el tipo "amarilla" lograron niveles muy buenos de micorrización. El género Rhizopogon posee una gran amplitud ecológica, apareciendo tanto en bosques jóvenes como maduros, y en hábitats diversos (Molina et al. 1999). Los hongos de los géneros Suillus y Amphinema, el "micelio blanco", "simple", "micelio marrón" y "tipo Tuber" siempre se expresaron en niveles inferiores al $10 \%$. El género Suillus varía en abundancia e importancia en las comunidades ectomicorrícicas, dependiendo del estadío sucesional del bosque (Dahlberg y Finlay 1999). Amphinema byssoides (Pers.) J. Erikss. tiene afinidad con plántulas, siendo muy frecuente y distribuida en viveros del oeste de Estados Unidos, y común también en bosques boreales maduros (Castellano y Molina 1989, Erland y Taylor 1999). El "tipo Tuber", de confirmarse su identidad genérica, ha sido reportado asociado tanto a árboles maduros (Fogel 1976, Hunt y Trappe 1987, Amaranthus et al. 1994, Colgan et al. 1999, Peay et al. 2007), a plántulas de bosques naturales (Cline et al. 2005, Walker et al. 2005), como a plántulas inoculadas artificialmente (Pacioni y Comandini 1999).

Contrariamente a lo expuesto por Smith y Read (2008), quienes plantean que los hongos de "etapa-temprana" tendrían un rol predominante en plántulas de viveros, en este trabajo se encontraron hongos micorrícicos "multi-etapa" colonizando las plántulas, probablemente por el origen fortuito del inóculo.

\section{CONCLUSIONES}

Se evidenció que el sistema productivo analizado incorpora fortuitamente inóculo micorrícico a la producción de plántulas, que dicho inóculo es viable y coloniza rápidamente a las plántulas inmediatamente después de suspendido el efecto de la fertirrigación, demostrando que la hipótesis de que las plántulas de pino ponderosa pueden establecerse y crecer satisfactoriamente en ambientes anectotróficos sin asociaciones EM es falsa. Asimismo, quedó evidenciado que el fertirriego tendría un efecto inhibidor en el establecimiento de la simbiosis, que comienza a producirse luego de suspendido éste.

\section{AGRADECIMIENTOS}

Al Ingeniero Agrónomo Juan Enricci por la información brindada y por permitir el uso de plántulas del vivero del PAIDER Forestal de la Universidad Nacional de la Patagonia San Juan Bosco. Al Sr. Vargas por permitir el uso de su campo para la instalación del ensayo. MESS, MR y CB son Becaria e Investigadores del CONICET, respectivamente.

\section{REFERENCIAS}

Agerer R ed. 1994. Colour Atlas of Ectomycorrhizae. Munich, Germany. Einhorn-Verlag.

Agerer R. 2001. Exploration types of ectomycorrhizae: a proposal to classify ectomycorrhizal mycelial systems according to their atterns of differentiation and putative ecological importance. Mycorrhiza 11: 107-114.

Amaranthus M, JM Trappe, L Bednar, D Arthur. 1994. Hypogeous fungal production in mature Douglas-fir forest fragments and surrounding plantations and its relation to coarse woody debris and animal mycophagy. Can. J. For. Res. 24: 2157-2165.

Barroetaveña C. 2004. Estudio de las ectomicorrizas de plantines y plantaciones de pino ponderosa (Pinus ponderosa Doug. ex Laws.) y pino oregón [Pseudotsuga menziesii (Mirb) Franco] en la región Andino Patagónica. Tesis Doctoral. San Carlos de Bariloche, Argentina. Universidad Nacional del Comahue. 225 p.

Barroetaveña C, M Rajchenberg, E Cázares. 2005. Mycorrhizal fungi in Pinus ponderosa introduced in Central Patagonia (Argentina). Nova Hedw 80: 453-464.

Barroetaveña C, E Cázares, M Rajchenberg. 2007. Ectomycorrhizal fungi associated with ponderosa pine and Douglas-fir: a comparison of species richness in nativewestern North American forests and Patagonian Plantations from Argentina. Mycorrhiza 17: 355-373.

Brundrett M, N Bougher, T Grove, N Malajczuk. 1996. Working with mycorrhizas in forestry and agriculture. Monograph 32. Canberra, Australia. Australian Center for International Agricultural Research. 374 p.

Castellano M, R Molina. 1989. Mycorrhizae. In Landis T, R Tinus, S Mc Donald, J Barnett eds. The Container Tree Nursery Manual. vol. 5. Agric. Handbook 674. Washington DC, USA. USDA-Forest Service. p. 101-167. 
Chakravarty P, L Chatarpaul. 1990. Effect of fertilization on seedling growth, ectomycorrhizal symbiosis, and nutrient uptake in Larix laricina. Can. J. For. Res. 20: 245-248.

Cline ET, JF Ammirati, RL Edmonds. 2005. Does proximity to mature trees influence ectomycorrhizal fungus communities of Douglas-fir seedlings? New Phytologist 166: 993-1009.

Colgan III W, AB Carey, JM Trappe, R Molina, D Thysell. 1999. Diversity and productivity of hypogeous fungal sporocarps in a variably thinned Douglas-fir forest. Can J For Res 29: 1259-1268.

Dahlberg A, RD Finlay. 1999. Suillus. In Cairney JWG, SM Chambers ed. Ectomycorrhizal fungi: key genera in profile. Berlin, Alemania. Springer. p. 33-64.

Danielson RM, S Vissar, D Parkinson. 1984. The effectiveness of mycelial slurries of mycorrhizal fungi for the inoculation of container-grown jack pine seedlings. Can. J. Res. 14: $140-142$.

Danielson RM. 1991. Temporal changes and effects of amendments on the occurrence of sheating (ecto-) mycorrhizas of conifers growing in oil sands tailings and coal spoil. Agric. Ecosyst. Environ. 35: 216-281.

Erland S, AFS Taylor. 1999. Resupinate ectomycorrhizal Fungi. In Cairney JWG, SM Chambers eds. Ectomycorrhizae fungi: key genera in profile. Berlin, Alemania. Springer. p. 347-364.

Fontenla S, R Godoy, P Rosso, M Havrylenko. 1998. Root associations in Austrocedrus forests and seasonal dynamics of arbuscular mycorrhizas. Mycorrhiza 8: 29-33.

Fontenla S, O Puntieri, JA Ocampo. 2001. Mycorrhizal associations in the Patagonian steppe, Argentine. Plant and Soil 232: 13-29.

Fogel R. 1976. Ecological studies of hypogeous fungi II: Basidiome phenology in a western Oregon Douglas-fir stand. Can. $J$ Bot 54: 1152-1162.

Gagnon J, CG Langlois, JA Fortin. 1987. Growth of containerized jack pine seedlings inoculated with different ectomycorrhizal fungi under controlled fertilization schedule. Can. J. For. Res. 17: 840-845.

Gagnon J, CG Langlois, JA Fortin. 1988. Growth and ectomycorrhiza formation of containerized black spruce seedlings as affected by nitrogen fertilization, inoculum type, and symbiont. Can. J. For. Res. 18: 922-929.

Godoy R, R Romero, R Carrillo. 1994. Estatus micotrófico de la flora vascular en bosques de coníferas del sur de Chile. Rev. Chilena Hist. Natural 67: 209-220.

Goodman DM, DM Durall, JA Trofymow eds. 1996. Concise descriptions of North American Ectomycorrhizae. Mycologue Publications \& -B.C. Forest Res. Dev. Agreement. Victoria. Canada. Canadian Forest Service.

Hunt GA. 1988. Effect of controlled-release fertilizers on formation of mycorrhizae and growth of container-grown Engelmann spruce. Proceedings of the Combined Western Forest Nursery Council and Intermountain Nursery Association meeting. Research Note RM-167. USDA Forest Service. 8 p.

Hunt GA, JM Trappe. 1987. Seasonal hypogeous sporocarp production in a western Oregon Douglas-fir stand. Can. J. Bot. 65: 438-445.

Jumpponen A. 2001. Dark septate endophytes-are they mycorrhizal? Mycorrhiza 11: 207-211.

Khasa PD, L Sigler, P Chakravarty, BP Dancik, L Erickson, D Mc Curdy. 2001. Effect of fertilization on growth and ectomycorrhizal development of container-grown and bareroot nursery conifer seedling. New Forests 22: 179-197.

Krasowski M, J Owens, L Tackaberry, H Massicotte. 1999. Above- and below-ground growth of white spruce seedlings with roots divided into different substrates with or without controlled release fertilizer. Plant Soil 217:131-143

Le Tacon F, D Mousain, J Garbaye, D Bouchard, JL Churin, C Argillier, JM Amirault, B Genere. 1997. Mycorhizes, pépinières et plantations forestières en France. Rev. For. Fr. 49: 131-154.

Martínez DB, C Barroetaveña, M Rajchenberg. 2007. Influencia del régimen de fertilización y del momento de inoculación en la micorrización de Pinus ponderosa en la etapa de vivero. Bosque 28(3): 226-233.

Menkis A, R Vasiliauskas, AFS Taylor, J Stenlid, R Finlay. 2005. Fungal communities in mycorrhizal roots of conifer seedlings in forest nurseries under different cultivation systems, assessed by morphotyping, direct sequencing and mycelial isolation. Mycorrhiza 16: 33-41.

Meyer FH. 1973. Distribution of ectomycorrhiza in native and man made forest. In Marks GC, TT Kozlowsky eds. Ectomycorrhizae, their ecology and physiology. New York, USA. Academic Press. p. 87-105.

Mikola P. 1988. Ectendomycorrhiza of conifers. Silva Fenn. 22: 19-27.

Molina R, J Chamard. 1983. Use of the ectomycorrhizal fungus Laccaria laccata in forestry II: Effects of fertilizer forms and levels on ectomycorrhizal development and growth of container-grown Douglas-fir and ponderosa pine seedlings. Can. J. Res. 13: 89-95.

Molina R, JM Trappe, LC Grubisha, JW Spatafora. 1999. Rhizopogon. In Cairney JWG, SM Chambers eds. Ectomycorrhizal fungi: key genera in profile. Berlin, Alemania. Springer. p. 129-186.

Ortega U, M Dunabeitia, S Menéndez, C González-Murúa, J Majada. 2004. Effectiveness of mycorrhizal inoculation in the nursery on growth and water relations of Pinus radiata in different water regimes. Tree Physiol. 24: 65-73.

Pacioni G, O Comandini. 1999. Tuber. In Cairney JWG, SM Chambers ed. Ectomycorrhizal fungi: key genera in profile. Berlin, Germany. Springer. p. 163-186.

Peay KG, TD Bruns, PG Kennedy, SE Bergemann, M Garbelotto. 2007. A strong species-area relationship for eukaryotic soil microbes: island size matters for ectomycorrhizal fungi. Ecology Letters 10: 470-480.

Read DJ, DH Lewis, AH Fitter, IJ Alexander eds. 1992. Mycorrhizas in ecosystems. Oxford, U.K. CAB International. 448 p.

Reitveld WJ, RA Sharp, MF Kienzler, RK Dixon. 1989. Development of ectomycorrhizae on container-grown European larch. Tree Plant Notes 70: 12-17.

Simard SW, MD Jones, DM Durral. 2002. Carbon and nutrient fluxes within and between mycorrhizal plants. In van der Heijden MGA, I Sanders eds. Mycorrhizal ecology. Berlin, Germany. Springer. p 34-74.

Smith SE, DJ Read. 1997. Mycorrhizal symbiosis. Cambridge, U.K. $2^{\text {nd }}$ ed. Academic Press. 605 p.

Smith SE, DJ Read. 2008. Mycorrhizal symbiosis. Cambridge, U.K. $3^{\text {th }}$ ed. Academic Press. 605 p.

Steel RGD, JH Torrie. 1988. Bioestadística: principios y procedimientos. New York, EE.UU. McGraw-Hill. 622 p. 
Stenström E, M Ek, T Unestam. 1990. Variation in field response of Pinus sylvestris to nursery inoculation with four different ectomycorrhizal fungi. Can. J. For. Res. 20: 1796-1803.

Stenström E, E Damm, T Unestam. 1997. Le role des mycorhizes dans la protection des arbres forestiers contre les agents pathogenes du sol. Rev. For. Fr. 49: 121-128.

Trappe JM 1977. Selection of fungi for ectomycorrhizal inoculation in nurseries. Annu. Rev. Phytopathol. 15: 203-222.
Van Tichelen KK, JV Colpaert, J Vangronsveld. 2001. Ectomycorrhizal protection of Pinus sylvestris against copper toxicity. New Phytol. 150: 203-213.

Walker JF, OK Miller, JL Horton. 2005. Hyperdiversity of ectomycorrhizal fungus assemblages on oak seedlings in mixed forests in the southern Appalachian Mountains. Molecular Ecology 14: 829-838.

Yu TEJ-C, KN Egger, RL Peterson. 2001. Ectomycorrhizal associations-characteristics and functions. Mycorrhiza 11: 167-177.

Recibido: 27.02 .09

Aceptado: 08.06.09 